PROCEEDINGS OF THE

AMERICAN MATHEMATICAL SOCIETY

Volume 134, Number 3, Pages 793-804

S 0002-9939(05)08006-8

Article electronically published on July 21, 2005

\title{
MONOTONICITY AND EXISTENCE OF PERIODIC ORBITS FOR PROJECTED DYNAMICAL SYSTEMS ON HILBERT SPACES
}

\author{
MONICA-GABRIELA COJOCARU
}

(Communicated by Carmen C. Chicone)

\begin{abstract}
We present here results about the existence of periodic orbits for projected dynamical systems (PDS) under Minty-Browder monotonicity conditions. The results are formulated in the general context of a Hilbert space of arbitrary (finite or infinite) dimension. The existence of periodic orbits for such PDS is deduced by means of nonlinear analysis, using a fixed point approach. It is also shown how occurrence of periodic orbits is intimately related to that of critical points (equilibria) of a PDS in certain cases.
\end{abstract}

\section{INTRODUCTION}

The theoretical study of projected differential equations (PrDE) and projected dynamical systems (PDS) started in the early 90s, in Euclidean space, with the papers [D-I] and [D-N], although similar concepts existed in the literature in [He], $\mathrm{Co}$ and $\mathrm{A}-\mathrm{C}$. Further papers and books continued the finite-dimensional study of this topic and explored its applicative potential (see [N-Z3, [N-S, $\mathbf{N - Z 1}$, [N-D-Z, [N-T-Z1], [Z-N2], Z-N1], D-Z-N], C-D-N] and the references therein).

The notion of PrDE was extended to infinite-dimensional spaces in $\mathrm{A}-\mathrm{C}$, Is-C3, [s-C2 , Coj1, C-J]. In Coj1, C-J], solutions were shown to exist for any PrDE on a Hilbert space and an infinite-dimensional PDS was defined and studied.

The original motivation for the theoretical study of a projected dynamics is found in finite-dimensional equilibrium problems (spatial price, oligopoly or traffic networks and financial equilibrium problems, just to name a few; see [N-S], [N-Z3], [N-D-Z], [N-T-Z1, [C-D-N]), although now infinite-dimensional models of PDS are coming to the forefront as in [C-D-N], Coj2. A study of existence for critical points (equilibria) of PDS and their stability properties was conducted in order to describe the behaviour of systems around steady states. In particular, the study used conditions of Minty-Browder monotonicity (see [N-Z1, [N-Z3], Is-C3, Is-C2], Coj1).

Received by the editors August 12, 2004 and, in revised form, October 6, 2004 and October $18,2004$.

2000 Mathematics Subject Classification. Primary 34A36, 34C25, 49J40; Secondary 37N40, $34 \mathrm{~A} 60$.

This research was funded by NSERC Discovery Grant No. 045997.

(C)2005 American Mathematical Society Reverts to public domain 28 years from publication 
In our pursuit to conduct a thorough theoretical study of projected dynamics, we ask here for the first time the question of existence of periodic orbits. The presence, or absence, of periodicity constitutes important information in the overall study of these dynamical systems and their applications. When periodicity is present, the underlying problem displays repetitive cycles, in which case a stability analysis of these cycles would be of interest. When there is no periodicity, the problem may evolve towards an equilibrium (if one exists) without being "trapped" in repetitive behaviour.

Under monotonicity conditions, we obtain the first results regarding periodicity for PDS. The paper is organized as follows: Section 2 gives a brief overview of the notions of PrDE and PDS. Sections 3 and 4 present the existence results for periodic cycles of a PDS and the relation between these and its critical points. We close with a few conclusions and acknowledgements.

\section{Preliminaries}

We assume the reader is familiar with the concepts of convex cone, polar cone and set-valued mapping. Let $X$ be a Hilbert space of arbitrary (finite or infinite) dimension. We do not assume separability of $X$. Let $K \subset X$ be a nonempty, closed and convex subset. Recall that for each $x \in K$ the set $T_{K}(x)=\overline{\bigcup_{h>0} \frac{1}{h}(K-x)}$ is the tangent cone to $K$ at $x$. The normal cone to $K$ at $x$ is the polar cone of $T_{K}(x)$, given by $N_{K}(x):=\left\{p \in X \mid\left\langle p, x-x^{\prime}\right\rangle \geq 0, \forall x^{\prime} \in K\right\}$.

Also recall that for any $z \in X$, there exists a unique element in $K$, denoted by $P_{K}(z)$, such that $\left\|P_{K}(z)-z\right\|=\inf _{x \in K}\|x-z\|$. This defines a mapping $P_{K}: X \rightarrow$ $K$ given by $z \mapsto P_{K}(z)$, called the projection operator of the space $X$ onto the subset $K$. The properties of the projection operator on Hilbert spaces are well known (see [Za ). Evidently, $P_{K}(x)=x$, for any $x \in K$. The Gateaux directional derivative of $P_{K}$ is defined as follows (for a proof see $\mathrm{Za}$, Lemma 4.6, or $\underline{\mathrm{Sh}}$, Section 3).

Proposition 2.1. For any $x \in K$ and any element $v \in X$ the limit

$$
\Pi_{K}(x, v):=\lim _{\delta \rightarrow 0^{+}} \frac{P_{K}(x+\delta v)-x}{\delta}
$$

exists and $\Pi_{K}(x, v)=P_{T_{K}(x)}(v)$.

Let $\Pi_{K}: K \times X \rightarrow X$ be the operator given by $(x, v) \mapsto \Pi_{K}(x, v)$. Note that, for each $x \in K$ and $v \in X$, there exists $n \in N_{K}(x)$ so that $\Pi_{K}(x, v)=v-n$, whenever $v \notin T_{K}(x)$; in other words, $\Pi_{K}$ is discontinuous on the boundary of the set $K$. In fact, $n:=P_{N_{K}(x)}(v)$, which can easily be seen from the classical Moreau's Theorem (see [C-J], Theorem 2.1, for the statement of this result), since $T_{K}(x)$ and $N_{K}(x)$ are nonempty, closed, convex cones polar to each other. Moreover $n$ as above is unique (from the general definition of the projection operator on a Hilbert space, given that $N_{K}(x) \subset X$ is nonempty, closed and convex). In this paper we frequently use the fact that for each $x \in K, v \in X$, there is an element $n \in N_{K}(x)$ as above, so that $\Pi_{K}(x, v)=v-n$.

Definition 2.1. Let $X$ be a Hilbert space of arbitrary dimension and let $K \subset X$ be a nonempty, closed and convex subset. Let $F: K \rightarrow X$ be a vector field. The 
discontinuous ordinary differential equation

$$
\frac{d x(t)}{d t}=\Pi_{K}(x(t),-F(x(t)))
$$

is called the projected differential equation associated with $-F$ and $K$.

A PrDE is a special case of the differential inclusions in [He, $\mathrm{Co}$ ] and $\mathrm{A}-\mathrm{C}$ ], Chapter 5 , Section 6 , where $\Pi_{K}$ is replaced by the set-valued mapping $x \mapsto-F(x)-$ $N_{K}(x)$ from $K$ to the subsets of $X$. The first formulation of equation (1), in the form given above, appeared in [D-I] with $X:=\mathbb{R}^{n}$ and $K$ a convex polyhedral set. It has been used, in the same finite-dimensional context, in [N-Z1], [N-Z3], [N-S], [N-T-Z1], N-D-Z], Z-N2 and more. It was extended to infinite-dimensional Hilbert spaces in [s-C3, Is-C2, Coj1, C-J].

Let $A C([0, \infty), K)$ denote the class of absolutely continuous functions defined on $[0, \infty)$ with values in $K$, which satisfy (11) for almost all $t \in[0, \infty)$. In [C-J], Theorem 3.1, and then in Coj1, Chapter 6, Theorem 6.1, it is shown that a PrDE has solutions in this class. For completeness, we give the statement of the result.

Theorem 2.1. Let $X$ be a Hilbert space of arbitrary dimension and let $K \subset X$ be a nonempty, closed and convex subset. Let $F: K \rightarrow X$ be a Lipschitz continuous vector field on $K$, and $x_{0} \in K$. Then the initial value problem

$$
\frac{d x(t)}{d t}=\Pi_{K}\left(x(t),-F(x(t)), x(0)=x_{0} \in K\right.
$$

has a unique solution in $A C([0, \infty), K)$.

Definition 2.2. A projected dynamical system is given by a mapping $\Phi$ : $R_{+} \times K \rightarrow K$ which solves the initial value problem:

$$
\dot{\Phi}(t, x)=\Pi_{K}(\Phi(t, x),-F(\Phi(t, x))), \quad \Phi(0, x)=x \in K .
$$

For the rest of the paper we denote by $x(t):=\Phi(t, x)$ the solution of (2) starting at the initial point $x \in K$. With this convention we define the following mapping: $K \longrightarrow A C([0, \infty), K)$, given by $x \mapsto x(t)$. Suppose now that $T \in(0, \infty)$ is given. We then define the mapping

$$
Q^{T}: K \longrightarrow K \text {, given by } x \mapsto Q^{T}(x)=x(T)=x+\int_{0}^{T} \Pi_{K}(x(s),-F(x(s)) d s .
$$

Our stated goal, that of finding periodic orbits of a PDS, is the same as solving the problem

$$
\frac{d x(t)}{d t}=\Pi_{K}(x(t),-F(x(t)), x(0)=x(T) \in K .
$$

However, this problem is equivalent to showing that the mapping $Q^{T}$ constructed above has a fixed point, which in turn is equivalent to showing that the functional equation $\left(i d_{K}-Q^{T}\right)(x)=0$ has at least one solution in $K$, where $i d_{K}$ is the identity map of $K$.

\section{Monotonicity And EXistence of Periodic orbits}

In this section we concentrate on showing that the functional equation $\left(i d_{K}-Q^{T}\right)(x)=0$ has at least one solution in $K$. We start by recalling the notions of Minty-Browder monotonicity and strict monotonicity, which are widely used in 
the theory of variational inequalities, discontinuous differential equations and functional equations (see [St], [Mi], $\mathrm{K}-\mathrm{S}], \mathrm{K}-\mathrm{Z}], \mathrm{B}-\mathrm{C}], \mathrm{A}-\mathrm{C}], \mathrm{Ka}],[\mathrm{Hk}],[\mathrm{Is}-\mathrm{C} 1]$ ).

Definition 3.1. Let $E$ be a Banach space, $E^{*}$ its dual and $S \subset X$ a nonempty subset. The mapping $f: S \rightarrow E^{*}$ is called monotone in the sense of MintyBrowder if, for any $x, y \in S$, we have $\langle f(x)-f(y), x-y\rangle \geq 0$, where $\langle\cdot, \cdot\rangle$ is the pairing on $E$. The mapping $f$ is called strictly monotone if, for any $x \neq y \in S$, we have $\langle f(x)-f(y), x-y\rangle>0$.

Monotonicity can also be defined for set-valued mappings ( $\mathrm{A}-\mathrm{C}]$, Chapter 3, Section 1, Definition 1): a set-valued map $A: S \rightarrow 2^{X}$, where $S \subseteq X$ is the domain of $A$ and $2^{X}$ is the power set of $X$, is called monotone if for any $x, y \in S$ and any $u \in A(x), v \in A(y)$, we have that $\langle u-v, x-y\rangle \geq 0$. In particular, we have the following result ([C-J], Lemma 2.1).

Lemma 3.1. The set-valued mapping $x \mapsto N_{K}(x)$ is monotone.

The next two theorems are very important preliminary results.

Theorem 3.1. Let $X$ be a Hilbert space, let $K \subset X$ be a nonempty, closed and convex subset, let $F: K \rightarrow X$ be a Lipschitz continuous vector field on $K$, and let $T>0$ be arbitrarily fixed. Then the mappings $Q^{T}: K \rightarrow K$ and $i d_{K}-Q^{T}: K \rightarrow X$ are continuous.

Proof. Let $\left\{x_{k}\right\}_{k \geq 0} \in K$ such that $x_{k} \rightarrow z$ as $k \rightarrow \infty$. As $K$ is closed, then $z \in K$. Let $x_{k}(t)$ and $z(t)$ be the solutions of (2) starting at $x_{k}$ and $z$ respectively. To complete the proof, it is enough to show that $Q^{T}: K \rightarrow K$ is continuous. We evaluate

$$
\begin{aligned}
& \frac{d}{d t}\left[\frac{1}{2}\left\|x_{k}(t)-z(t)\right\|^{2}\right]=\left\langle\frac{d}{d t}\left[x_{k}(t)-z(t)\right], x_{k}(t)-z(t)\right\rangle \\
= & \left\langle\Pi_{K}\left(x_{k}(t),-F\left(x_{k}(t)\right)\right)-\Pi_{K}(z(t),-F(z(t))), x_{k}(t)-z(t)\right\rangle \\
= & \left\langle-F\left(x_{k}(t)\right)-n_{x_{k}(t)}+F(z(t))+n_{z(t)}, x_{k}(t)-z(t)\right\rangle .
\end{aligned}
$$

Using Lemma 3.1, we obtain

$$
\frac{d}{d t}\left[\frac{1}{2}\left\|x_{k}(t)-z(t)\right\|^{2}\right] \leq\left\langle-F\left(x_{k}(t)\right)+F(z(t)), x_{k}(t)-z(t)\right\rangle .
$$

Suppose $b$ is the Lipschitz constant of $F$. Then

$$
\frac{d}{d t}\left[\frac{1}{2}\left\|x_{k}(t)-z(t)\right\|^{2}\right] \leq b\left\|x_{k}(t)-z(t)\right\|^{2} .
$$

Let $\phi^{k}(t):=\left\|x_{k}(t)-z(t)\right\|$. The above relation can be written as

$$
\phi^{k}(t) \frac{d \phi^{k}(t)}{d t} \leq b\left(\phi^{k}(t)\right)^{2} \text {, or equivalently } \phi^{k}(t) \frac{d \phi^{k}(t)}{d t}-b\left(\phi^{k}(t)\right)^{2} \leq 0 .
$$

We multiply the last inequality with the positive number $e^{-2 b t}$ and, keeping in mind that $\frac{d}{d t}\left[\left(\phi^{k}(t)\right)^{2} e^{-2 b t}\right]=2 e^{-2 b t}\left[\phi^{k}(t) \frac{d \phi^{k}(t)}{d t}-b\left(\phi^{k}(t)\right)^{2}\right]$, we obtain

$$
\frac{d}{d t}\left[\phi^{k}(t)^{2} e^{-2 b t}\right] \leq 0 .
$$

Integrating between 0 and $T$, we have

$$
\left(\phi^{k}(T)\right)^{2} \leq 2 e^{2 b T}\left\|x_{k}-z\right\|^{2} .
$$

As $x_{k} \rightarrow z$, then $\phi^{k}(T) \rightarrow 0$ as $k \rightarrow \infty$ and the proof is complete. Evidently, the mapping $i d_{K}-Q^{T}$ is also continuous. 
Suppose now that the vector field $F$ in (2) is monotone in the sense of Definition 3.1. The following result holds.

Theorem 3.2. Let $X$ be a Hilbert space and $K \subset X$ a nonempty, closed and convex subset. Let $F: K \rightarrow X$ be a monotone, Lipschitz continuous vector field on $K$, and $T>0$ arbitrarily fixed. Then the mapping $i d_{K}-Q^{T}: K \rightarrow X$ is monotone.

Moreover, if $F$ is strictly monotone, then $i d_{K}-Q^{T}$ is also strictly monotone.

Proof. Let $x, y \in K$ be arbitrarily fixed and let $x(t), y(t)$ be the solutions of (2) starting at $x$ and $y$ respectively. We evaluate

$$
\begin{gathered}
\frac{d}{d t}[\langle-x(t)+y(t), x-y\rangle]=\langle-\dot{x}(t)+\dot{y}(t), x-y\rangle \\
=\langle-\dot{x}(t)+\dot{y}(t), x(t)-y(t)\rangle+\langle-\dot{x}(t)+\dot{y}(t), x-x(t)-y+y(t)\rangle .
\end{gathered}
$$

We evaluate further the last two terms of the above relation:

$$
\begin{gathered}
\langle-\dot{x}(t)+\dot{y}(t), x(t)-y(t)\rangle=\left\langle-\Pi_{K}(x(t),-F(x(t)))+\Pi_{K}(y(t),-F(y(t))), x(t)-y(t)\right\rangle \\
=\langle F(x(t))-F(y(t)), x(t)-y(t)\rangle+\left\langle n_{x(t)}-n_{y(t)}, x(t)-y(t)\right\rangle .
\end{gathered}
$$

Keeping in mind Lemma 3.1 and the fact that $F$ is monotone we obtain

$$
\langle-\dot{x}(t)+\dot{y}(t), x(t)-y(t) \geq 0 \text {. }
$$

On the other hand,

$$
\langle-\dot{x}(t)+\dot{y}(t), x-x(t)-y+y(t)\rangle=\frac{d}{d t}\left[\frac{1}{2}\|x-x(t)-y+y(t)\|^{2}\right] .
$$

Going back to our initial computation we thus have

$$
\frac{d}{d t}[\langle-x(t)+y(t), x-y\rangle] \geq \frac{d}{d t}\left[\frac{1}{2}\|x-x(t)+y(t)-y\|^{2}\right] .
$$

But

$$
\int_{0}^{T} \frac{d}{d t}\left[\frac{1}{2}\|x-x(t)+y(t)-y\|^{2}\right] d t=\frac{1}{2}\|x-x(T)+y(T)-y\|^{2}
$$

and integrating the last inequality from 0 to $T$ we have

$$
\left\langle\left(i d_{K}-Q^{T}\right)(x)-\left(i d_{K}-Q^{T}\right)(y), x-y\right\rangle \geq 0 .
$$

Evidently, if $F$ is strictly monotone, then inequality (4) is strict. This further implies that inequality (6) is strict and the proof is complete.

Theorem 3.3 below is based on [K-Z], Chapter 5, Section 42, Theorem 42.6, which we give here for completeness: Let $E$ be a reflexive Banach space with dual $E^{*}$, and let $f: E \rightarrow E^{*}$ be a monotone mapping that maps each strongly convergent sequence in $E$ into a weakly convergent sequence in $E^{*}$. If for some $r>0$, we have $\langle f(x), x\rangle \geq 0$, for each $\|x\|=r$, then the equation $f(x)=0$ has at least one solution in the ball $\{x \mid\|x\| \leq r\}$.

We prove that a similar result holds on a subset of a Hilbert space $X$.

Theorem 3.3. Let $X$ be a Hilbert space and $K \subset X$ a nonempty, closed, convex subset with $0 \in \operatorname{int}(K)$. Let $f: K \rightarrow X$ be a monotone mapping which maps strongly convergent sequences in $K$ into weakly convergent sequences in $X$. If there exists $r>0$ such that $\overline{B(0, r)} \subseteq K$ with the property that $\langle f(x), x\rangle \geq 0$, for each $\|x\|=r$, then the equation $f(x)=0$ has at least one solution in the set $\overline{B(0, r)} \subseteq K$.

Moreover, if $f$ is strictly monotone, then the solution is unique. 
Proof. Let $x \in K$ be arbitrarily fixed. We define $U(x):=\{y \in \overline{B(0, r)} \mid\langle f(x)$, $x-y\rangle \geq 0\}$. This set is weakly compact. We want to show that any finite collection of such sets intersect. If this is true, then the collection of weakly closed sets $U(x)$ has the finite intersection property and hence there exists a common element, say $y^{*} \in \bigcap_{x \in K} U(x)$.

Let us prove the finite intersection property of the sets $U(x)$. Consider $\left\{x_{1}, \ldots\right.$, $\left.x_{m}\right\} \in K$ and their corresponding sets $U\left(x_{i}\right), i \in\{1, \ldots, m\}$. Let $E_{0}$ be the linear space spanned by $\left\{x_{1}, \ldots, x_{m}\right\}$, let $\left\{z_{1}, \ldots, z_{n}\right\}$ be a basis of $E_{0}$ and consider the set $E_{0} \cap K$. This intersection set is not empty. We define the mapping $G: E_{0} \cap$ $K \rightarrow X, G(x)=\sum_{j=1}^{n}\left\langle f(x), z_{j}\right\rangle z_{j}$. This mapping is continuous as $f$ maps strongly convergent sequences into weakly convergent ones.

Following an argument similar to that in the proof of [K-Z], Chapter 5, Section 42 , Theorem 42.6 , we can conclude that the equation $G(x)=0$ has a solution on the ball $\left\{x \in E_{0} \cap K \mid\|x\| \leq r\right\}$. Let $x_{0}$ be such a solution. Then $\sum_{j=1}^{n}\left\langle f\left(x_{0}\right), z_{j}\right\rangle z_{j}=0$ implies $\left\langle f\left(x_{0}\right), z_{j}\right\rangle=0$ since $\left\{z_{1}, \ldots, z_{n}\right\}$ is a basis of $E_{0}$; thus $\left\langle f\left(x_{0}\right), p\right\rangle=0$ for any $p \in E_{0}$. In particular, if $p:=x_{i}-x_{0}$, for an arbitrarily fixed $i \in\{1, \ldots, m\}$, we obtain $\left\langle f\left(x_{0}\right), x_{i}-x_{0}\right\rangle=0$. From here $\left\langle f\left(x_{i}\right), x_{i}-x_{0}\right\rangle=\left\langle f\left(x_{i}\right)-f\left(x_{0}\right), x_{i}-x_{0}\right\rangle \geq 0$, based on the monotonicity of $f$ and therefore $x_{0} \in \bigcap_{i=1}^{m} U\left(x_{i}\right)$. Thus the collection $U(x)$ has the finite intersection property.

Let us denote by $y^{*}$ the common point of the sets $U(x)$. It follows that $\langle f(x)$, $\left.x-y^{*}\right\rangle \geq 0$ for all $x \in K$. As $y^{*} \in \overline{B(0, r)} \subseteq K$, then two cases arise: $y^{*} \in$ $\overline{B(0, r)} \cap \operatorname{int}(K)$ or $y^{*} \in \overline{B(0, r)} \cap \partial K$. Consider $\left\{t_{k}\right\}_{k \geq 0}$ a sequence of positive reals, such that $t_{k} \rightarrow 0$ as $k \rightarrow \infty$.

Case 1. For any $h \in X$, there exists $k_{0}>0$ such that for any $k \geq k_{0}, x:=$ $y^{*}+t_{k} h \in K$. Then $\left\langle f\left(y^{*}+t_{k} h\right), h\right\rangle \geq 0$, and as $t_{k} \rightarrow 0,\left\langle f\left(y^{*}\right), h\right\rangle \geq 0$, for any $h \in X$; thus $f\left(y^{*}\right)=0$ and the proof is complete in this case.

Case 2. For any $\delta \in(0,1)$ arbitrarily fixed, the point $\delta y^{*} \in \overline{B(0, r)} \cap \operatorname{int}(K)$. From Case 1 we have $\left\langle f\left(\delta y^{*}\right), h\right\rangle \geq 0$, for any $h \in X$. Choose $\left\{\delta_{k}\right\}_{k \geq 0} \in(0,1)$ such that $\delta_{k} \rightarrow 1$. Then $\left\langle f\left(\delta_{k} y^{*}\right), h\right\rangle \geq 0$. As $f$ is strongly to weakly continuous, we obtain $\left\langle f\left(y^{*}\right), h\right\rangle \geq 0$, for any $h \in X$. This again implies $f\left(y^{*}\right)=0$, and the existence part of the proof is complete.

Now assume that there are $y_{1}^{*} \neq y_{2}^{*}$, solutions of $f(x)=0$. From here and the strict monotonicity of $f$ we have that $0=\left\langle f\left(y_{1}^{*}\right)-f\left(y_{2}^{*}\right), y_{1}^{*}-y_{2}^{*}\right\rangle>0$, which is a contradiction. Thus if $f$ is strictly monotone, then there is a unique solution for $f(x)=0$.

Remark 3.1. The assumption $\overline{B(0, r)} \subseteq K$ cannot be replaced with $\overline{B(0, r)} \cap K$ in Theorem 3.3. Otherwise, suppose $K:=[-0.5, \infty) \subset \mathbb{R}$ and $f: \mathbb{R} \rightarrow \mathbb{R}$ is given by $f(x)=1+x$. This is a closed, convex set with $0 \in \operatorname{int}(K)$ and $f$ is a monotone, continuous mapping. If we let $r:=2$, then there exists $B(0,2)$ such that $\overline{B(0,2)} \cap K \neq \emptyset$ and $\langle f(x), x\rangle \geq 0$, for $x \in\{-2,2\}$. However, the only solution of $f(x)=0$ does not belong to $K$. 
Putting together Theorems 3.1, 3.2 and 3.3, we obtain

Theorem 3.4. Let $X$ be a Hilbert space, $K \subset X$ a nonempty, closed and convex subset with $0 \in \operatorname{int}(K)$ and $F: K \rightarrow X$ a monotone, Lipschitz continuous vector field on $K$. If there exist $r>0$ and $T>0$ with the properties: $\overline{B(0, r)} \subseteq K$ and $\left\langle\left(i d_{K}-Q^{T}\right)(x), x\right\rangle \geq 0$ for all $x \in \partial \overline{B(0, r)}$, then there exists a point $x^{*} \in \overline{B(0, r)} \subseteq$ $K$ such that $x^{*}(T)=x^{*}$ (i.e., a fixed point of $Q^{T}$ or equivalently, a solution of (3) ).

Moreover, if $F$ is strictly monotone, for each $r, T>0$ with the above property, there exists a unique fixed point of the corresponding mapping $Q^{T}$.

Proof. For $T>0$ as in the hypothesis we can construct the mapping $Q^{T}$. Theorem 3.1 shows that the mapping $i d_{K}-Q^{T}$ is continuous, whereas Theorem 3.2 shows that $i d_{K}-Q^{T}$ is monotone. This means that $i d_{K}-Q^{T}$ satisfies the assumptions of Theorem 3.3 and the conclusion follows. Finally, if $F$ is strictly monotone, from Theorem $3.2, i d_{K}-Q^{T}$ is strictly monotone and again by Theorem 3.3 the conclusion follows.

In the introductory section we mentioned that the initial motivation behind the study of a PDS resided in equilibrium problems. Hence the existence study of critical points (or equilibria) of PDS and their properties was extremely important, as they correspond to equilibrium states of the underlying applied problem.

In general, an equilibrium $x$ of a PDS is characterized by the condition $\Pi_{K}(x,-F(x))=0$, or equivalently, $-F(x) \in N_{K}(x)$. The existence of such points was established via the theory of variational inequalities, in the sense that

$\left(^{*}\right)$ the equilibria of a PDS coincide with the solutions to the variational inequality (VI):

$$
\text { find } x \in K \text { such that }\langle F(x), y-x\rangle \geq 0 \text {, for all } y \in K
$$

(see [D-N] for $X:=R^{n}$ and Is-C3, Proposition 6, or [C-J], Theorem 2.2, for infinite-dimensional Hilbert spaces). We note here that the relation between PDS and VI justifies the convention of using $-F$ in the definition of a PrDE/PDS, whereas $F$ is used to define the associated VI.

However, it is obvious that any equilibrium of $\operatorname{PDS}$ has the property $Q^{T}(x)=x$, for any $T>0$; thus it is a fixed point for the mapping $Q^{T}$, for any $T>0$. In its present form, Theorem 3.4 does not distinguish between a fixed point of $Q^{T}$ arising from an equilibrium of the PDS and a fixed point of $Q^{T}$ arising from a periodic cycle. Therefore we need

Corollary 3.4.1. Under the hypotheses of Theorem 3.4 the following statements hold:

1) a fixed point $x^{*}$ of the mapping $Q^{T}$ is an equilibrium of (2) if $-F\left(x^{*}\right) \in$ $N_{K}\left(x^{*}\right)$;

2) a fixed point of the mapping $Q^{T}$ is a solution of (3) if $-F\left(x^{*}\right) \notin N_{K}\left(x^{*}\right)$.

\section{Strict monotonicity, EQUilibria AND PERIOdic orbits of PDS}

In this section we continue our discussion of equilibrium points and periodic orbits for a PDS given by

$$
\frac{d x(t)}{d t}=\Pi_{K}(x(t),-F(x(t)))
$$

where this time $F: K \rightarrow X$ is strictly monotone. In general, the following result holds (proved in [N-D-Z], Proposition 2, for Euclidean space). 
Lemma 4.1. Let $X$ be a Hilbert space, $K \subset X$ a nonempty, closed and convex subset and $F: K \rightarrow X$ strictly monotone. Then (7) has at most one equilibrium.

Proof. Suppose there exist at least two equilibrium points of (7), $x_{1} \neq x_{2} \in K$, so that $-F\left(x_{i}\right) \in N_{K}\left(x_{i}\right)$, for $i \in\{1,2\}$. From Lemma 3.1, the monotonicity of the mapping $x \mapsto N_{K}(x)$ implies that

$$
\left\langle-F\left(x_{1}\right)+F\left(x_{2}\right), x_{1}-x_{2}\right\rangle \geq 0 .
$$

But the strict monotonicity of the mapping $F$ implies that

$$
\left\langle-F\left(x_{1}\right)+F\left(x_{2}\right), x_{1}-x_{2}\right\rangle<0,
$$

which is a contradiction. Therefore there exists at most one equilibrium of (7).

Let $x^{*}$ be an equilibrium of (77). In [N-Z1, Theorem 4.2 (where $X:=\mathbb{R}^{n}$ and $K$ is a convex polyhedron), it is shown that $F$ strictly monotone on $K$ implies $x^{*}$ is a global strict monotone attractor, i.e., is such that for any solution $x(t)$ of (17) starting at $x(0)=x \in K$, the function $t \mapsto\left\|x(t)-x^{*}\right\|$ is decreasing. We show that this stays true in our more general context.

Lemma 4.2. Let $X$ be a Hilbert space, $K \subset X$ a nonempty closed, convex subset and $F: K \rightarrow X$ a strictly monotone Lipschitz continuous vector field on $K$. If $x^{*}$ is an equilibrium of (7), then it is a global strict monotone attractor.

Proof. We have that

$$
\frac{d}{d t}\left(\frac{1}{2}\left\|x(t)-x^{*}\right\|^{2}\right)=\left\langle x(t)-x^{*}, \frac{d x(t)}{d t}\right\rangle=\left\langle x(t)-x^{*},-F(x(t))-n\right\rangle,
$$

where $n \in N_{K}(x(t))$. Now $\left\langle x(t)-x^{*},-n\right\rangle \leq 0$ from the definition of $N_{K}(x(t))$ and so

$$
\frac{d}{d t}\left(\frac{1}{2}\left\|x(t)-x^{*}\right\|^{2} \leq\left\langle x(t)-x^{*},-F(x(t))\right\rangle .\right.
$$

Since $x^{*}$ is an equilibrium of (7), then $-F\left(x^{*}\right) \in N_{K}\left(x^{*}\right)$. From here we have $\left\langle x(t)-x^{*},-F\left(x^{*}\right)\right\rangle \leq 0$. Since $F$ is strictly monotone, $\left\langle x(t)-x^{*}, F\left(x^{*}\right)-F(x(t))\right\rangle<$ $0 \Longrightarrow\left\langle x(t)-x^{*},-F(x(t))\right\rangle\left\langle\left\langle x(t)-x^{*},-F\left(x^{*}\right)\right\rangle \leq 0\right.$. Based on (8) , the conclusion follows.

We are now ready to prove the following result.

Theorem 4.1. Let $X$ be a Hilbert space, $K \subset X$ a nonempty closed, convex subset and $F: K \rightarrow X$ a strictly monotone Lipschitz continuous vector field on $K$. Then the following hold:

1) if there exists an equilibrium of (7), there are no periodic cycles for the underlying PDS.

2) if $K$ is compact, there are no periodic cycles for the underlying PDS.

Proof. 1) Let us assume that there exists at least one periodic cycle of (7) of period $T>0$; hence $x(t)=x(t+T)$, for any $t>0$. Let $x^{*}$ be the unique (by Lemma 4.1) strict monotone attracting (by Lemma 4.2) equilibrium of (7). Then as $T<t+T$ we have $\left\|x(t+T)-x^{*}\right\|<\left\|x(t)-x^{*}\right\|$, but this is a contradiction with $x(t)=x(t+T)$.

2) For convex, compact sets $K \subset X$, the VI problem of finding $x \in K$ so that $\langle F(x), y-x\rangle \geq 0$ for any $y \in K$, is always solvable ([Mi], Theorem 1 ). Hence, by the relation between VI and PDS described in Section 3 in $\left(^{*}\right)$, the PDS has at least one equilibrium. As $F$ is strictly monotone, the equilibrium is unique and according to 1 ) above there are no periodic cycles. 
Remark 4.1. 1) For closed, convex sets $K \subset X$ with $0 \in \operatorname{int}(K)$, Theorem 4.1 can be alternatively proved by noting that the unique equilibrium of (7) is also the unique fixed point of any mapping $Q^{T}$ (Theorem 3.4); hence periodic cycles cannot form.

2) Theorem 4.1 is directly applicable to examples of PDS such as the ones in Z-N1], Example 1, Z-N2], Example 5.1, and [N-D-Z, Section 2, Example 2, where the constraint sets are usually $\mathbb{R}_{+}^{n}$, as well as to the ones in [C-D-N].

Theorem 3.4 and Theorem 4.1 lead to the following concluding remark.

Remark 4.2. Let $X$ be a Hilbert space, $K \subset X$ a nonempty, closed and convex subset with $0 \in \operatorname{int}(K)$ and $F: K \rightarrow X$ a Lipschitz continuous vector field on $K$ which is monotone, but not strictly monotone. If there exist $r, T>0$ as in Theorem 3.4 such that the mapping $Q^{T}$ has fixed points, then both equilibria and periodic orbits can occur and Corollary 3.4.1 is in effect.

Here is an example to illustrate the last remark.

Example. Let $K:=[-\infty, 1.2] \times[-0.5, \infty] \in \mathbb{R}^{2}$ and $F(x, y)=(y,-x+0.5)$. The vector field $F$ is Lipschitz continuous and monotone, but not strictly monotone, as $\langle F(x, y)-F(a, b),(x-a, y-b)\rangle=0$ for any pair of points $(x, y),(a, b) \in \mathbb{R}^{2}$. The $\mathrm{PrDE}$ in this case has the form

$$
\frac{d(x(t), y(t))}{d t}=\Pi_{K}((x(t), y(t)),(-y(t), x(t)-0.5)),
$$

and the point $(0.5,0)$ is an equilibrium.

Now let $r:=0.2$ and consider the ball $\overline{B((0,0), 0.2)} \subset K$. Let $T:=2 \pi$. We claim that for each $(x, y) \in \partial \overline{B((0,0), 0.2)}$ we have $\langle(x, y)-(x(2 \pi), y(2 \pi)),(x, y)\rangle \geq 0$. Indeed, given the set $K$ and the definition of $\Pi_{K}$ as the projection of $-F$ on the tangent cone at any $(x, y) \in K$, we obtain

$$
\Pi_{K}((x, y),(-y, x-0.5)):=\left\{\begin{array}{cc}
(-y, x-0.5) & \text { if } y \geq-0.5 \text { and } 0<x<1.2 \\
(-y,-0.5) & \text { if } y \geq-0.5 \text { and } x \leq 0 .
\end{array}\right.
$$

Now we see that any solution of (9) starting in the region $\partial \overline{B((0,0), 0.2)} \cap\{y \geq$ -0.5 and $0<x<1.2\}$ is the same as the solution of

$$
\frac{d(x(t), y(t))}{d t}=(-y(t), x(t)-0.5)
$$

starting in the same region. Such a solution is given by $x(t)=C_{2} \sin (t)+C_{1} \cos (t)+$ $0.5, y(t)=-C_{2} \cos (t)+C_{1} \sin (t)$, and obviously $\langle(x, y)-(x(2 \pi), y(2 \pi)),(x, y)\rangle \geq$ 0 , where $(x, y)=(x(0), y(0))$. For a trajectory of (9) starting in the region $\partial \overline{B((0,0), 0.2)} \cap\{y \geq-0.5$ and $x \leq 0\}$, and having a discontinuous velocity, we compute1 1 approximates on the time interval $[0,2 \pi]$, following the constructive proof of Theorem 3.1 in [C-J]. We see that the condition $\langle(x, y)-(x(2 \pi), y(2 \pi)),(x, y)\rangle \geq 0$ holds for each of these approximates: Figure 1 (left) depicts such an approximate (with constant step 0.02) for the trajectory of (9) starting at the point $(-0.2,0)$. Since the sequence of approximates converges to the sought trajectory, then our claim is true.

\footnotetext{
${ }^{1}$ All computations and figures were done with MAPLE 8.
} 

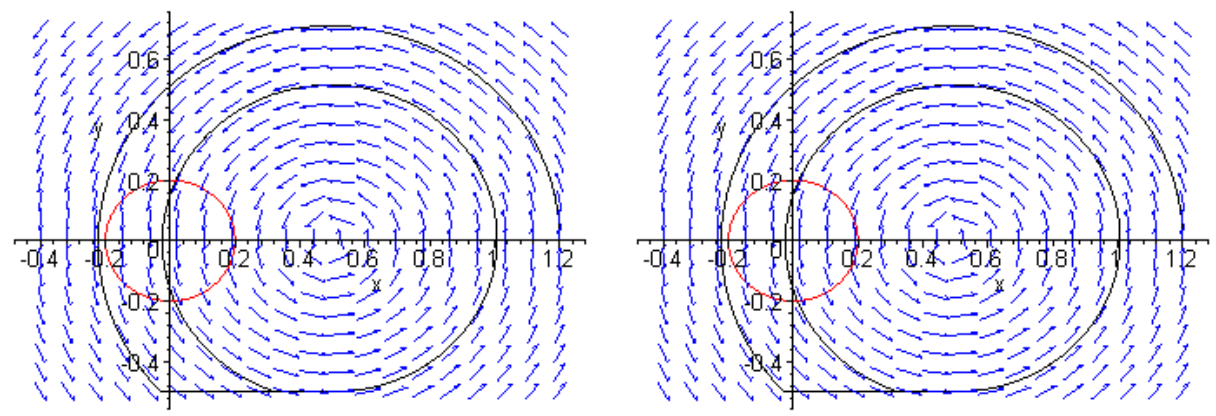

Figure 1.

Therefore, following Theorem 3.4, there exists at least one fixed point of the mapping $Q^{2 \pi}$ in the ball $\overline{B((0,0), 0.2)}$. In fact, see Figure 1 (right), all points of $\overline{B((0,0), 0.2)}$ within the disk of radius 0.5 centered at $(0.5,0)$ are periodic with period $2 \pi$. All other points of $\overline{B((0,0), 0.2)}$ are not periodic; the trajectories starting at these points (for example $(1.2,0)$ ) are eventually trapped on the periodic cycle centered at $(0.5,0)$ of radius 0.5 .

\section{Conclusions AND ACKNOWLEDGEMENTS}

The present work constitutes the beginning of the study of periodic behaviour for PDS. Theorem 3.4 relies on continuity and monotonicity, concepts used in the theory of PDS known to date. In particular, monotonicity conditions have been employed to show both existence and stability properties of equilibria. Theorem 4.1 shows that periodic behaviour for systems under strict monotonicity is not possible. However, there are systems where periodic behaviour takes place (as our example shows); therefore its study in relation to equilibrium states, or in relation to stability concepts, comes to the forefront. New results in this direction are obtained and constitute the topic of future work.

There are at least a few immediate open questions that arise from this work, of which we mention here two:

(i) do periodic cycles for PDS exist in the absence of monotonicity conditions?

(ii) do periodic cycles for PDS exist over constraint sets that do not satisfy the condition $0 \in \operatorname{int}(K)$ ?

It is our belief that these questions can lead to new and exciting opportunities for theoretical results as well as interesting consequences for applications.

The author would like to thank Dr. L. Jonker for useful discussions on the topic, which helped the presentation of this work. The author also thanks the referee for the careful examination of this work and for readability suggestions.

\section{REFERENCES}

[A-C] Aubin, J. P. and Cellina, A., Differential Inclusions, Springer-Verlag, Berlin (1984). MR0755330 (85j:49010)

[B-C] Baiocchi, C. and Capelo, A., Variational and Quasivariational Inequalities. Applications to Free Boundary Problems., J. Wiley and Sons, (1984). MR0745619 (86e:49018)

[Coj1] Cojocaru, M. G., Projected Dynamical Systems on Hilbert Spaces, Ph. D. Thesis, Queen's University, (2002). 
[Coj2] Cojocaru, M. G., Infinite-dimensional projected dynamics and the 1-dimensional obstacle problem, to appear in J. Funct. Spaces Appl. (2005).

[C-J] Cojocaru, M. G. and Jonker, L. B., Projected differential equations in Hilbert spaces, Proc. Amer. Math. Soc., Volume 132, Number 1, 183-193(2004). MR2021261 (2004k:34011)

[C-D-N] Cojocaru, M. G., Daniele, P. and Nagurney, A., Projected dynamical systems and evolutionary (time-dependent) variational inequalities on Hilbert spaces with applications, to appear in J. Optim. Theory Appl. (2005).

[Co] Cornet, B., Existence of Slow Solutions for a Class of Differential Inclusions, J. Math. Anal. Appl. 96 (1983), 130-147. MR0717499 (86a:34029)

[D-Z-N] Dong, J., Zhang, D. and Nagurney, A., A projected dynamical system model of general financial equilibrium with Stability Analysis, Math. Comput. Modelling, Vol. 24, No. 2(1996), 35-444. MR1403528

[D-I] Dupuis, P. and Ishii, H., On Lipschitz continuity of the solution mapping to the Skorokhod problem, with applications, Stochastics Stochastics Rep., Vol. 35(1990), 31-62. MR.1110990 (93e:60110)

[D-N] Dupuis, P. and Nagurney, A., Dynamical systems and variational inequalities, Ann. Oper. Res. 44, (1993), 9-42. MR1246835 (94k:49009)

[He] Henry, C., An existence theorem for a class of diffferential equations with multivalued right hand sides, J. Math. Anal. Appl. 41 (1973), 179-186. MR0335906 (49:684)

[Hk] Heikkilaa, S., Monotonone Iterative Techniques for Discontinuous Nonlinear Differential Equations, Monographs and Textbooks in Pure and Applied Mathematics, 181, M. Dekker (1994).

[Is-C1] Isac, G. and Cojocaru, M. G., Functions without exceptional family of elements and the solvability of variational inequalities on unbounded sets, Topol. Methods Nonlinear Anal., Vol. 20 (2002), 375-391. MR1962226 (2003k:49017)

[Is-C2] Isac, G. and Cojocaru, M. G., Variational inequalities, complementarity problems and pseudo-monotonicity. Dynamical aspects., in "Seminar on fixed point theory ClujNapoca", Proceedings of the International Conference on Nonlinear Operators, Differential Equations and Applications, Babes-Bolyai University of Cluj-Napoca, Vol. III(2002), 41-62. MR1929747 (2003k:49018)

[Is-C3] Isac, G. and Cojocaru, M. G., The projection operator in a Hilbert space and its directional derivative. Consequences for the theory of projected dynamical systems, J. Func. Spaces Appl., Vol. 2, Number 1 (2004), 71-95. MR2028181 (2004m:34136)

[Ka] Karamardian, S. and Schaible, S., Seven kinds of monotone maps, J. Optim. Theory Appl., Vol. 66, No. 1(1990), 37-46. MR.1061909 (91e:26016)

[K-S] Kinderlehrer, D. and Stampacchia, G., An Introduction to Variational Inequalities and Their Applications, Academic Press (1980). MR0567696 (81g:49013)

[K-Z] Krasnoselskii, M. A. and Zabreiko, P. P., Geometrical Methods of Nonlinear Analysis, Springer-Verlag, A Series of Comprehensive Studies in Mathematics, Vol. 263(1984). MR0736839 (85b:47057)

[Mi] Minty, G. J., On variational inequalities for monotone operators, Adv. in Math. 30(1978), 1-7. MR0511738 (80c:47045)

[N-Z1] Nagurney, A. and Zhang, D., On the Stability of Projected Dynamical Systems, J. Optim. Theory Appl. 85 (1995), 97-124. MR1330844 (96f:34066)

[N-Z3] Nagurney, A. and Zhang, D., Projected Dynamical Systems and Variational Inequalities with Applications, Kluwer Academic Publishers (1996).

[N-S] Nagurney, A. and Siokos, S., Financial Networks: Statics and Dynamics, SpringerVerlag (1997).

[N-D-Z] Nagurney, A., Dupuis, P. and Zhang, D., A Dynamical Systems Approach for Network Oligopolies and Variational Inequalities, Ann. Regional Science 28 (1994), 263-283.

[N-T-Z1] Nagurney, A., Takayama, T. and Zhang, D., Projected dynamical systems modelling and computation of spatial networks equilibria, Networks, Vol. 26(1995), 69-85.

[Sh] Shapiro, A. S., Existence and differentiability of metric projections in Hilbert spaces, SIAM J. Optim. 4(1994), 130-141. MR1260410 (94m:90111)

[St] Stampacchia, G. Variational Inequalities, Theory and Applications of Monotone Operators, Proc. NATO Advanced Study Institute, Venice 1968, Ed. Oderisi, Gubbio, Italy (1969), 101-192. MR0425699 (54:13652) 
[Za] Zarantonello, E., Projections on convex sets in Hilbert space and spectral theory, Contributions to Nonlinear Functional Analysis, Publ. No. 27, Math. Res. Center, Univ. Wisconsin, Academic Press (1971), 237-424. MR0388177 (52:9014) MR0388178 (52:9015)

[Z-N1] Zhang, D. and Nagurney, A., Stability analysis of an adjustment process for oligopolistic market equilibrium modeled as a projected dynamical system, Optimization, Vol. 36, No. 2(1996), 263-285. MR:1419267

[Z-N2] Zhang, D. and Nagurney, A., Formulation, stability and computation of traffic network equilibria as projected dynamical systems, J. Optim. Theory Appl., Vol. 93, No. 2(1997), 417-444. MR.1448567 (98b:90161)

Department of Mathematics and Statistics, MacNaughton Hall, Room 548, UniverSity of Guelph, Guelph, Ontario, Canada N1G 2W1

E-mail address: mcojocar@uoguelph.ca 